\title{
Evaluation of adhesion strength of protective coatings with Al powder by adhesion work of initial suspension towards metal surface
}

\author{
Natalia Antonova ${ }^{1, *}$ \\ ${ }^{1}$ Kamensk Institute (Affiliation) of SRSTU (NPI) named after M.I. Platov, 23 K. Marx Ave, \\ Kamensk-Shakhtinsky, 347800, Russia
}

\begin{abstract}
The influence of energetic factors upon surface properties of suspensions on the basis of $\mathrm{Na}-\mathrm{CMC}$ with aluminum powder, used to produce anticorrosion coatings, is revealed. It is shown that relative adhesion work, got by measuring the extent of suspensions surface strain and the angles of wetting the steel surface with suspensions, correlate with adhesion strength of coatings containing aluminum powder.
\end{abstract}

\section{Introduction}

Ecologically clean materials having given functions, productions of which is based upon the renewable plant material, attract researchers' uprising interest. The polymer Nacarboxymethyl cellulose (Na-CMC) of plant origin is being widely used. It is used as a solutions stabilizer in oil production [1], as a binding agent in medicine [2-4], as a binding component of lithium-ion battery anode [5-7] in energy production. Water solutions of the polymer are a cheap medium for creating functional nano-sized additives, synthesized from metal powders [8]. Thus, polymer Na-CMC is used mostly as a composition component or solution stabilizer.

Earlier we showed the possibility to use Na-CMC polymer as matrixes to form functional coatings: porous, protecting, antifriction [9-11]. Anticorrosion coatings [10], produced from polymer suspensions $\mathrm{Na}-\mathrm{CMC}$ with aluminum powder, are used in chemical production to protect steel tanks from aggressive non-water media. In such a composite the polymer is stable towards aggressive non-water media as aluminum powder raises the mechanical and adhesion strength of the coating. The mechanical strength of coatings amounts 10-20 MPa [12] and is rather typical for films based on cellulose ethers [13]. Adhesion strength of coatings, created from suspensions with metal powders, to a great extent depends upon initial compositions rheology, metal powders dispersibility and micro particles distribution within the area of coating contact with protecting surface. The evaluation of mechanical, physical and special properties of coatings is made at the stage of final works and being unsatisfactory leads to the necessity of correcting and reprocessing which is economically unfavorable. Stating the influence of fillings upon the strength of adhesion between the substrate and the suspension on the stage of suspension production

* Corresponding author: melnik1@,rambler.ru 
lets reduce time losses and predict adhesion strength of coatings. In the paper presented the correlation between surface properties of suspension and adhesion strength of anticorrosion coating is revealed.

The purpose of the paper is to determine the influence of energy factors upon the surface properties of the Na-CMC suspension with aluminum powder and match these characteristics with adhesion strength of formed anticorrosion coatings.

\section{Materials and Methods}

Morphology of initial materials and coatings, created upon steel sbstrates, were examined with the help of electronically scanning microscope Quanta 200 in the spectrum of secondary and back-scattered electrons. Metal powder dispersibility was determined with the help of MicroTrac Bluewave analyzer. X-ray diffraction analysis (XDA) of films was done using diffractometer ARL X'TRA within the anglular spacing of $2 \theta$ from $5^{\circ}$ to $80^{\circ}$ with the interval of $0.020^{\circ}$ emission $-\mathrm{CuK}_{\alpha}, \mathrm{K}_{\beta}$. The analyses were made using equipment of CUC Nanotechnologies, SRSTU (NPI) named after M.I. Platov. Organic component of composites and polymer powders infrared spectra (IR-spectra) was registered by spectrophotometer Varian Excalibur 3100 FT-IR (CUC SFU), within the spacing of $600 \div 4000 \mathrm{~cm}^{-1}$.

Purified powder of Na-CMC with polymerization degree 450, flexibilizer glycerin, powder of spherical disperse aluminum ASD-1 were used in the course of research. Images of powders are presented in fig. 1, a-b. Na-CMC polymer has fiber structure, fibers in dry powder are in disorder, separate fragments are rolled into balls, the thickness if fibers reaches $14 \div 20 \mu \mathrm{m}$. A1 particles form is close to spherical, particles size is not bigger than $50 \mu \mathrm{m}$. Histogram (fig. 1, c) shows the results of aluminum powder disperse analysis. Aluminum powder contains submicron fraction $(\leq 0,1 \mu \mathrm{m})$, thin fraction with the size of $0,05 \div 0,06 \mu \mathrm{m}$ prevails. Particles with the size about $19 \mu \mathrm{m}$ make the peak value according to the histogram. The powder biggest particles have the size of $37 \div 44 \mu \mathrm{m}$, they make 4,5 $\%$ of overall amount.

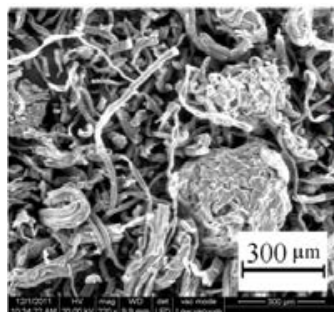

a)

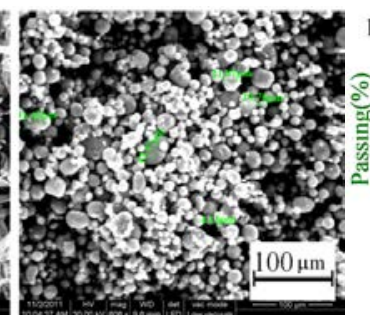

b)

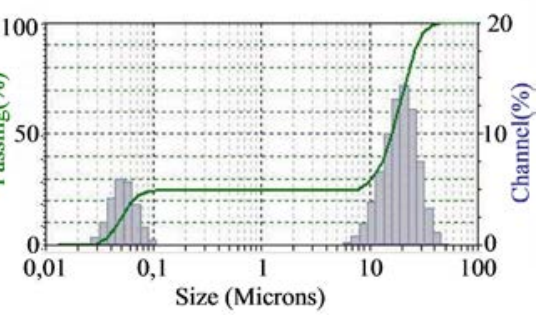

c)

Fig. 1. SEM-images of powders: polymer Na-CMC (a), aluminum (b); histogram of particles distribution according to their sizes (c)

Compositions for coatings forming were got by mixing gel-like $2,3 \%$ water solution of $\mathrm{Na}-\mathrm{CMC}$ powder with glycerin and aluminum powder particles under the temperature of $(25+1){ }^{\circ} \mathrm{C}$. Distilled water was used as dissolver. To reveal the influence of aluminum powder upon the surface properties of suspensions and coatings structure forming, samples from water polymer suspensions both with and without metal fillings were produced. Components combinations and temperature conditions of coatings production proposed in the paper [10] were used. Proportions of components in coatings compositions are presented in table 1 . Coatings were formed under the temperature of $(36 \pm 1){ }^{\circ} \mathrm{C}$. 
Table 1. Proportion of components in suspensions of Na-CMC water solutions

\begin{tabular}{|c|c|}
\hline $\begin{array}{l}\text { Composition } \\
\text { number }\end{array}$ & Proportion: Na-CMC+glycerin+Al, g \\
\hline 1 & $100,00+0+0$ \\
\hline 2 & $100,00+1,00+0$ \\
\hline 3 & $100+1,00+2,50$ \\
\hline
\end{tabular}

To explain the metal powder influence upon the coatings adhesive strength the surface properties of materials on the interface were determined: the solid (rimmed steel 08) - fluid (compositions 1-3). Good wetting on the solid-fluid interface is one of the conditions to get a hard composite material. The measure of wetting is the interfacial angle $\theta$ between the wetted surface (steel) and the fluid surface on the wetted perimeter. The $\theta$ value was determined with the help of projecting the «sitting drop» onto the screen under the temperature of $(36 \pm 1){ }^{\circ} \mathrm{C}$. The amount of wetted angle is an important but yet unsufficient parameter, characterising coating adhesion towards the steel substrate. That is why with the help of du-Nui circular method under the temperature of $(36 \pm 1){ }^{\circ} \mathrm{C}$ the surface strain $\sigma$ of $\mathrm{Na}-\mathrm{CMC}$ water solution and suspensions on its basis was determined. Distilled water was used as standard liquid. On the basis of the got amounts of $\theta$ and $\sigma_{1}$ the wetting energy was calculated:

$$
W_{E}=\sigma \cdot \cos \theta
$$

Adhesion work was calculated using the Young - Dupre equation:

$$
W_{A}=\sigma \cdot(1+\cos \theta)
$$

To evaluate the interaction of components in suspensions the cohesion work, which is determined by energy consumption for removable discontinuity with the cross-section equalling the unit area, was calculated. After the discontinuity the surface of two area units is formed and cohesion work makes :

$$
W_{C}=2 \sigma
$$

The process of wetting characterises the spreading coefficient S. Under full wetting $\mathrm{S} \rightarrow 0$. The spreading coefficient was calculated with the help of the following formula:

$$
S=W_{A}-W_{C}=\sigma \cdot(\cos \theta-1)
$$

The connection between adhesion and cohesion work is shown through the relative value of liquid adhesion work:

$$
Z_{A}=\frac{W_{A}}{W_{C}}
$$

The evaluation of adhesion strength of adhesion between the substrate and the coating was done with the help of "mushrooms" discontinuing method. The composition under research was spread onto the "mushrooms" - 08 rimmed steel samples with the diameter of about $(25,0 \pm 0,1) \mathrm{mm}$, the samples were connected and withstood in a thermo case within 6 hours under the temperature of $(36 \pm 1){ }^{\circ} \mathrm{C}$. The surface of samples was worked with abrasives and defatted with acetone. The trials were done with the help of tensile-testing 
machine TM-4 providing the movement speed of travelling gripper relative to fixed gripper of $2,5 \mathrm{~mm} / \mathrm{min}$ under the temperature of $(23 \pm 2){ }^{\circ} \mathrm{C}$.

\section{Results and Discussions}

Figure 2 shows images of coating compositions 1-3cross-sections. The film, made from Na$\mathrm{CMC}$ has both ordered and amorphous areas (fig. 3a). There are splits (imagefragment, fig. $3 b$ ), the film is breakable, it easily exfoliates from the steel substrate. Flexibilizer reduces strains in the polymer matrix not leading to noticeable improving the adhesion strength between the coating and the substrate (fig. 3c). Al particles are arranged within the matrix comparatively evenly (fig. 3d) which witnesses of sedimentation stability of initial suspension. Earlier, in paper [14], it was stated that adhesive strength between the substrate and the coating depends mostly upon the presence of particles whose size is compareable with substrate microrelief (for the used steel the particles size is $<10 \mu \mathrm{m}$ ). Filling substrate fine irregularities they serve as anchor elements, helping to increase surfaces adhesive strength.

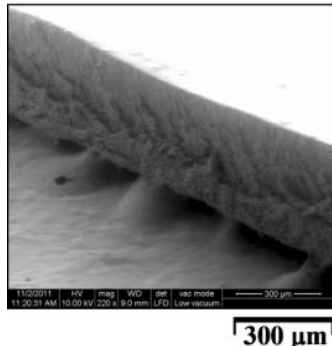

a)

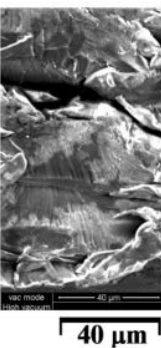

b)

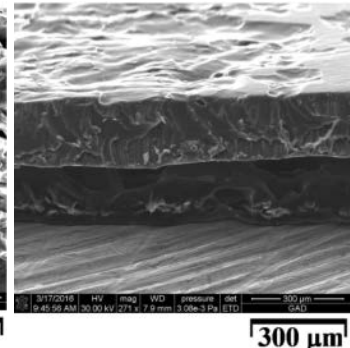

c)

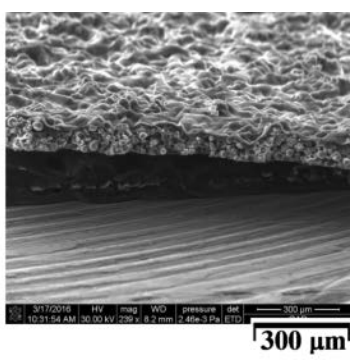

d)

Fig. 2. SEM-images of end surfaces of coatings compositions: 1 - (a, b); 2 - (c); 3 - (d)

The results of XDA (fig. 3) showed the presence of metal aluminum inside the coating (PDF card 000-04-0787). There are no aluminum oxides, what can be explained by their low contain in aluminum powder. Bragg angles18-20 area corresponds to initial cellulose crystallites [13].

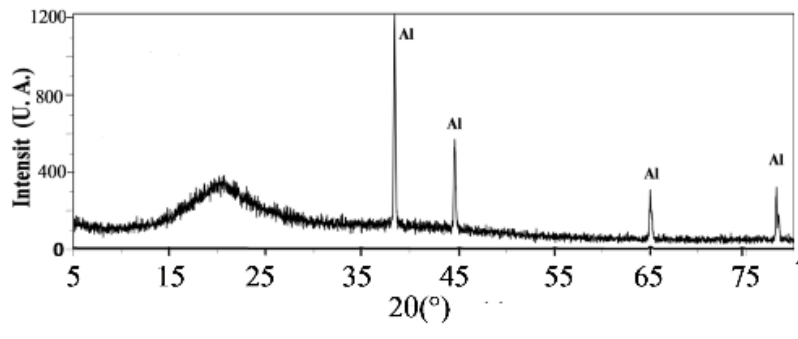

a)

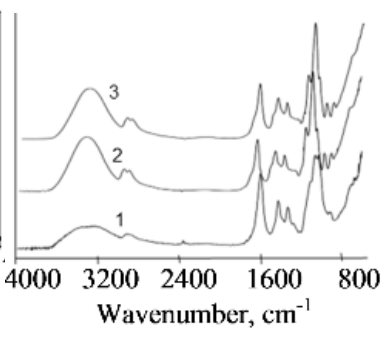

b)

Fig. 3. Difractogram of composition 3 coating - (a) ; IR-spectra of compositions 1-3 (b)

Research held with the help of IR- spectroscopy method speaks in favour of forming hydrogen links between polymer macromolecules and oxide shells of aluminum particles. There is an absorption stripe in $3350-3200 \mathrm{sm}^{-1}$ area in the spectra, which corresponds the absorption stripe of $\mathrm{OH}$-group valency fluctuations. This stripe intensity for the coating containing aluminum is much higher than for polymer films without a filling. This fact witnesses of strengthening the composite system structure which contains $\mathrm{Al}$ at the expense 
of hydrogen links, accessible to interact in $\mathrm{OH}$-groups of water Na-CMC solution. The $\mathrm{XDA}$ and IR spectroscopy results lead to a conclusion about the fact that chemical composition of coatings does not change in the process of forming; interaction between components is achieved at the expense of intermolecular hydrogen links.

Table 2 shows the results of examining surface properties of "steel substrate suspension" system. Histograms of fig. 4 represent amounts of suspension adhesion relative work towards steel substrates and got during the experiment amount of adhesion strength of coatings, made of suspensions.

Table 2. Surface characteristics of "steel substrate - suspension" system

\begin{tabular}{|l|l|l|l|l|l|l|}
\hline $\begin{array}{l}\text { Compostion } \\
\text { number }\end{array}$ & $\sigma, \mathrm{mN} / \mathrm{m}$ & $\theta,{ }^{0}$ & $W_{E}, \mathrm{~mJ} / \mathrm{m}^{2}$ & $W_{A}, \mathrm{~mJ} / \mathrm{m}^{2}$ & $W_{C}, \mathrm{~mJ} / \mathrm{m}^{2}$ & $S$ \\
\hline 1 & 127,4 & 23 & 117,6 & 245,0 & 254,8 & $-9,8$ \\
\hline 2 & 125,7 & 30 & 108,9 & 234,6 & 251,4 & $-16,8$ \\
\hline 3 & 120,1 & 14 & 114,5 & 236,6 & 240,2 & $-3,6$ \\
\hline & & & & & & \\
\hline
\end{tabular}

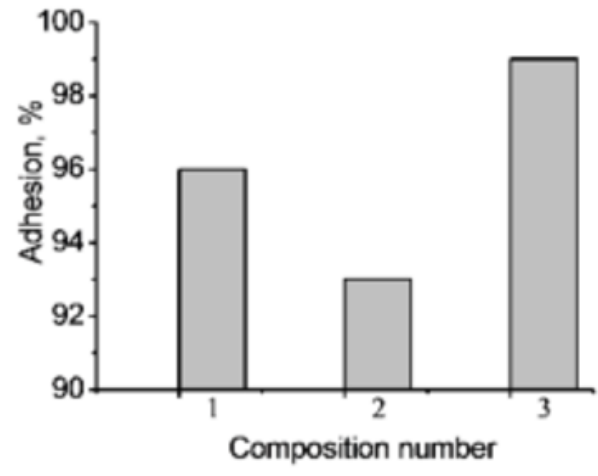

a)

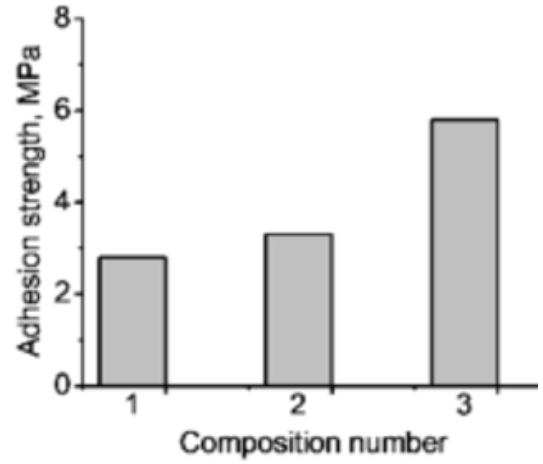

b)

Fig. 4. Influence of glycerin and aluminum powder upon: adhesion of suspensions with steel surface - (a); adhesion strength of coatings - (b)

Surface strain reduces when glycerin is added which is explained by forming monomolecular layers of boundary lubricant between polymer macromolecules. Al powder decreases surface strain. Al microparticles structurize the solution, reducing interaction between Na-CMC molecules and decreasing the strain. Wetting coefficient increases, reaching its maximum amount for composition 3 which contains glycerin and aluminum. Aluminum powder presence in compositions improves adhesion strength from 2,8 $\mathrm{MPa}$ to 5,8 $\mathrm{MPa}$ (fig. 4, b). Relative adhesion work for suspensions with aluminum powder, calculated with the help of Dupre-Young equation correlates with adhesion strength between coatings and steel substrate.

\section{Conclusion}

Adequacy between the values of adhesion work got from calculating the amount of suspensions surface strain and angles of wetting the steel substrate surface with suspensions is revealed. Adhesion work, calculated with the help of Dupre-Young equation, correlates with adhesion strength of coatings for compositions filled with aluminum powders. The proposed approach may be used as a basis of simple methods of preliminary evaluation of adhesion connections strength between coatings and protected surfaces. 


\section{References}

1. Aliu A. O., Guo J., Wang S., Zhao X., Journal of Natural Gas Science and Engineering. 32, 491-500 (2016)

2. K. Yamaguchi, M. Prabakaran, M. K. Xu Gang, Min Chung, In Chul Um, M. Gopiraman, I. S. Kim, Materials Letters. 168 (1), 56-61 (2016)

3. Shi D. D., Wang F. J., Lan T., Zhang Y. H., Shao Z. Q., Cellulose. 23 (3), 1899-1909 (2016)

4. Azzaoui K., Mejdoubi E., Lamhamdi A., Jodeh S., Hamed O., Berrabah M., Jerdioui S., Salghi R., Akartasse N., Errich A., Rios A., Zougagh M., Carbohydrate polymers. 167, 59-69 (2017). Cuesta N., Ramos A., Camean I., Antuna C., Garcia A. B., Electrochimica Acta. 155, 140-147 (2015)

5. K. KierzekJournal of Materials Engineering and Performance. 25 (6), 2326-2330 (2016)

6. Jeschull F., Lacey M.J., Brandell D., Electrochimica Acta. 175, 141-150 (2015)

7. Pomogajlo A.D., Rozenberg A.S. and Ufljand I.E. Metal nanoparticles in polymers. (Moscow: Chemistry, 2000)

8. Antonova N. M., Babichev A. P., Berezovsky V. S., Journal of Surface Investigation: X-ray, Synchrotron and Neutron Techniques. 11 (5), 955-959 (2017). DOI: 10.1134/S1027451017050032

9. Antonova N.M., Corrosion: materials, protection. 5, 35-41 (2012)

10. Antonova N., Lisnichenko I, Sibirka I. MATEC Web of Conferences. 2017. V. 129: International Conference on Modern Trends in Manufacturing Technologies and Equipment, ICMTMTE 2017; Sevastopol; Russian Federation; 11 September 2017 - 15 September 2017. - Article Number: 02008. - Режим доступа : https://www.matecconferences.org/articles/matecconf/pdf/2017/43/matecconf_icmtmte2017_02008.pdf DOI: $10.1051 /$ matecconf $/ 201712902008$

11. Antonova N.M., Russian Journal of Non-Ferrous Metals. 50 (4), 419-423, (2009)

12. Petropavlovsky G.A. Hydrophilous and partially substituted carboxethyl esters of cellulose and their modification by chemical crossing of cellulose. (St. Petersburg: Science, 1988)

13. Antonova N. M. , Kulinich V. I., Journal of Surface Investigation. X-ray, Synchrotron and Neutron Techniques. 2 (5), 744-747 (2008) 